\title{
Abordagem Cognitiva da Compreensão Leitora: implicações para a educação e prática clínica
}

\author{
Rosinda Martins Oliveira* \\ Universidade Federal do Rio de Janeiro, Rio de Janeiro, Brasil
}

\begin{abstract}
RESUMO
A importância da compreensão leitora é indiscutível e as publicações nacionais sobre o tema ainda ocorrem em número modesto, tendo em vista o desempenho dos brasileiros em termos desta habilidade. Este artigo apresenta a abordagem cognitiva da compreensão leitora. Enquanto que esta abordagem considera a participação de funções cognitivas de baixo e alto nível neste processo, de forma semelhante a outras teorias, delas se diferencia pela ênfase nas funções executivas e pelo detalhamento do processo de compreensão. Além disso, considera o standard de coerência do leitor como modulador desse processo. O modelo é apresentado e discutido em termos de suas implicações para a prática clínica e para a educação.
\end{abstract}

Palavras-chave: compreensão leitora; funções executivas; processos inferenciais.

\begin{abstract}
Cognitive view of reading comprehension

The importance of reading comprehension is indisputable. National publications on the subject still occur in very modest numbers, given the restriction shown by Brazilians in terms of this skill. This paper presents the cognitive view of reading comprehension. While this theory considers the participation of cognitive functions of lower and higher level in reading comprehension, similarly to other theoretical models, it is distinguished by an emphasis on the executive functions and by the attempt to detail the process of comprehension. It also considers the reader's standard of coherence as a modulator of this process. The model is presented and discussed in terms of its implications for clinical practice and education.
\end{abstract}

Keywords: reading comprehension; executive functions; inferencial processes.

\section{INTRODUÇÃO}

A compreensão é o propósito básico da leitura e é fundamental para o desenvolvimento cognitivo e acadêmico, visto ser instrumental para a aquisição de outros conhecimentos e habilidades. Apesar da relevância inegável da leitura, dados do Instituto Nacional de Estudos e Pesquisas Educacionais (INEP/MEC) $(2009 ; 2012)$ mostram a grande limitação do brasileiro para decifrar e compreender um texto escrito. Estudos básicos (Capellini, Santos, \& Uvo, 2015, Corso, Sperb, \&, Salles, 2013; Guimarães, Emmerick, Vicente, \& Soares, 2012; Machado, 2010; Oliveira, 2015; Parente, Holderbaum, Virbel, \& Nespoulous, 2005; Salles \& Parente, 2004; Spinillo \& Hodges, 2012, por exemplo) e aplicados (Capellini, Pinto \& Cunha, 2015; Ferreira \& Dias, 2002; Gomes \& Boruchovitch, 2011; Joly \& Piovezan, 2012; Santos \& Oliveira, 2010) tem sido feitos no Brasil, a respeito do desenvolvimento da leitura e de intervenções para melhoria da compreensão leitora, mas ainda em quantidade modesta.

Dentre as formulações teóricas que tentam dar conta dos processos cognitivos envolvidos na compreensão de um texto escrito (Kintsch \& van Dijk, 1978; Lorch \& van den Broek, 2000), está a Abordagem Cognitiva da Compreensão Leitora (Cognitive View of Reading Comprehension) (Kendeou, van den Broek, Helder \& Karlsson, 2014). Este modelo, como 
outros (por exemplo, the simple view of reading Gough \& Tunmer, 1986), propõe que processos cognitivos de baixo e alto nível estão envolvidos nesta habilidade, apontando a decodificação e vocabulário entre os primeiros. No entanto, se diferencia por tentar delinear de forma mais clara o papel de processos cognitivos de alto nível, como as funções executivas, na Compreensão. Este artigo tem por objetivo apresentar esta abordagem e discutir as suas implicações para a prática clínica e para a educação.

\section{COMPREENSÃO LEITORA}

A abordagem cognitiva da compreensao leitora (ACCL), de forma semelhante a outras formulações teóricas relativas ao tema (p.ex. Gough \& Tunmer, 1986), reconhece que a compreensão depende da operação conjunta e integrada de vários processos cognitivos, dentre eles: processar palavras individualmente, identificar e acessar suas representações mentais (fonológicas, ortográficas e semânticas), conectar estas representações, construindo a compreensão do sentido subjacente às sentenças e, finalmente, processar e conectar idéias para construir uma representação mental do texto, que possibilite a sua compreensão como um todo. Além disso, a ACCL ressalta que a compreensão depende também de características do leitor, de demandas específicas da tarefa e das propriedades do texto (Kendeou et al., 2014).

Assim, para que o leitor chegue a compreender o que lê, são necessários processos de baixo nível, sendo o mais importante deles a decodificação. Para compreender é preciso que o sujeito possa decifrar as palavras escritas, isto é, chegar a saber o seu significado, acessando, a partir da sequência de letras escritas, suas representações fonológicas, ortográficas e semânticas.

No entanto, estes processos não são ainda suficientes para a compreensão de um texto escrito. Esta requer a construção de uma representação mental coerente do texto que inclui, em um todo, as informações nele escritas, a associação entre partes da informação textual e entre estas e a base de conhecimentos que o sujeito adquiriu ao longo da vida. Estas associações, necessárias à compreensão, são feitas através de processos inferenciais passivos e ativos.

Neste ponto, a ACCL, dá um passo que a diferencia de outros modelos como, por exemplo, o Simple
View of Reading (Gough \& Tunmer, 1986), na medida em que afirma que os processos inferenciais recrutam a atenção, a memória de trabalho e de modo mais geral, as funções executivas, e ainda tenta explicitar como estes processos participam na compreensão.

Enquanto que a representação mental coerente do texto é o produto final da compreensão, é fundamental conhecer o processo de construção desta representação e que processos cognitivos estão envolvidos, visto ser aqui que podem incidir as intervenções que buscam melhorar esta habilidade (Rapp, Vander Broek, McMaster, Kendeou, \& Espin, 2007).

A ACCL chama ainda atenção para a influência do standard de coerêcia do leitor sobre a compreensão, isto é, o quanto ele tem por objetivo entender enquanto lê determinado texto.

\section{Processos de baixo nível envolvidos na compreensão leitora}

A necessidade da integridade, ou pelo menos de um bom funcionamento, dos processos de decodificação, para que se chegue a compreender o que se lê é inquestionável e é reconhecida por outros (p.ex. Simple view of reading, Gough \& Tunmer, 1986). Se o leitor decodifica as palavras de forma errada, os processos de mais alto nível já receberão as informações equivocadas, o que afetará a compreensão. E, mesmo que a dificuldade de decodificação não resulte em erro, a não automatização desse processo pode comprometer a compreensão, pois resultará no desvio de recursos atencionais que não mais estarão disponíveis integralmente para os processos de alto nível (Perfetti \& Hart, 2002). A dificuldade de compreensão apresentada por indivíduos com dislexia do desenvolvimento, pelo menos em parte, são devidas a mecanismos como estes (Gough \& Tunmer, 1986; Bishop \& Snowling, 2004).

\section{Processos cognitivos de alto nível envolvidos na compreensão leitora}

Kendeou e cols (2014) listaram entre os processos cognitivos de alto nível envolvidos na compreensão leitora, os processos inferenciais e as funções executivas (memória de trabalho, controle inibitório, planejamento e alocação de atenção), indicando as evidências empíricas dessa participação. Embora a participa- 
ção dos processos inferenciais e da memória de trabalho seja apontada em outros modelos como o Simple view of reading (Gough \& Tunmer, 1986), o mesmo não se aplica às outras funções executivas.

\section{Processos inferenciais}

A compreensão de um texto escrito requer não apenas a compreensão das idéias explicitamente escritas, mas também de idéias que são veiculadas no texto, de modo menos explicitado. Isto é alcançado através da capacidade de produzir inferências - de inferir ideias a partir da associação de duas ou mais partes do texto, ou dessas partes com o conhecimento prévio do leitor (Ackerman, 1988; Cain, Oakhill, \& Bryant, 2000).

Dentre os tipos de inferência estão as intra-textuais (que mantém a coerência textual) e as extra-textuais (elaborativas). Um exemplo de inferência intra-textual é visto na sentença "Pedro implorou a sua mãe que o deixasse ir à festa"; para compreendê-la é preciso perceber que o pronome "o" se refere a "Pedro". Na sentença "Katia deixou o vaso cair. Ela se apressou em pegar a vassoura e a pá para recolher os pedaços", é preciso que o leitor recorra à sua base de conhecimentos (inferência extra-textual), para perceber que o vaso quebrou. Esta informação não está explícita no texto, e é fundamental para estabelecer conexões entre as duas sentenças, possibilitando sua compreensão.

Existe consenso téorico e evidências empíricas de que a habilidade de gerar inferências é central para a compreensão leitora (Kintsch, 1998; Oakhill, 1994; van den Broek, 1994), havendo indicação de que esses processos são causa, e não consequêcia da compreensão (Cain e Oakhill, 1999). O comprometimento desta capacidade afeta diretamente a compreensão leitora (Magliano, Wiemer-Hastings, Millis, Munoz, \& McNamara, 2002; Oakhill \& Yuilll, 1996). De forma condizente, a estimulação da capacidade de fazer inferências resulta em melhoria da compreensão (Brown, Palincsar \& Armbuster, 1984; Hansen \& Pearson, 1983; Oliveira, 2015; Paris, Cross \& Lipson, 1984; Yuill \& Joscelyne, 1988).

No entanto, esta capacidade não é específica para a leitura. Ela também é requerida para a compreensão de discursos que chegam ao sujeito através de outras mídias, até mesmo visuais. O nível de desenvolvimento da habilidade de gerar inferências se mostra intere- lacionado em diferentes mídias como, por exemplo, uma história ouvida, vista na televisão ou lida. Além disso, quando avaliada aos 4 anos de idades (antes do aprendizado da leitura) esta capacidade foi preditora de seu nível de desenvolvimento aos 8 anos de idade, na leitura (Kendeou, Bohn-Gettler, White, \& Vanden Broek, 2008).

Estudos mostram que o aprimoramento da geração de inferências começa muito antes do aprendizado da leitura (Akerman, 1986; 1988). Ao longo do desenvolvimento, há um aumento na espontaneidade e iniciativa deste comportamento (Oakhill \& Bryant, 2003) e na quantidade das inferências. Ainda, progressivamente estas são geradas a partir da conexão de unidades cada vez maiores e mais distantes no texto (parágrafos, episódios, sessões) e há um incremento no seu grau de abstração (van den Broek, 1997).

A existência de uma capacidade de fazer inferências que está para além da leitura pode ajudar a entender os resultados de Clarke, Snowling, Truelove e Hulme (2010), que obtiveram melhor efeito sobre a compreensão leitora usando estimulação oral do que escrita, em um estudo que incluía protocolos de estimulação de construção de inferências.

A compreensão requer a participação de processos inferenciais automáticos e estratégicos. Os processos inferenciais automáticos não estão sob controle do leitor, e ocorrem sem esforço. O mais importante deles é a propagação de ativação. Os conceitos em uma sentença disparam a ativação de outros conceitos relacionados, ativados durante a construção da representação de partes anteriores do texto na base de conhecimentos do leitor (O'Brien \& Albrecht, 1992; Kintsh, 1988; van den Broek, Young, Tzeng, \& Linderholm, 2004). Os processos inferenciais estratégicos, por outro lado, requerem esforço e são recrutados a partir da demanda de estabelecimento de coerência do leitor, dependendo, assim, do controle executivo. Incluem, por exemplo, procurar ativamente informações em partes anteriores do texto e na base de conhecimentos, para explicar a informação em uma sentença que está sendo lida (van den Broek, 1994, 2010)

\section{Funções executivas}

Apesar da existência de vários modelos de funções executivas, de modo geral, estas podem ser definidas como processos que controlam e regulam o compor- 
tamento durante a execução de uma tarefa (Miyaki et al., 2000). São constituídas por um conjunto de processos top-down necessários quando (a) o sujeito não dispõe de programas de ação previamente construídos para dar conta de uma atividade (novidade) e (b) quando esta atividade recruta recursos atencionais (por sua relevância e complexidade). Requerem esforço cognitivo, isto é, o sujeito precisa manter o curso do comportamento apesar dos possíveis distratores representados, inclusive, por programas de ação ativados de forma automática pelos estímulos presentes (Diamond, 2013).

Kendeou et al. (2014) descreveram o papel da memória de trabalho, do controle inibitório e do planejamento, além da capacidade de alocação da atenção, na compreensão leitora.

\section{Memória de trabalho}

A memória de trabalho é definida como a capacidade de manter ativas na mente as informações, ao mesmo em que se trabalha com elas (Baddeley \& Hitch, 1994), e sua importância para a compreensão leitora é facilmente previsível. Para compreender uma sentença é preciso que o sujeito mantenha ativa a representação de seus elementos iniciais enquanto lê e representa o restante dela. Para estabelecer relações entre partes do texto, o sujeito precisa manter ativas representações relativas a elas enquanto processa as conexões. E ainda, para estabelecer inferências extratextuais, o sujeito precisa manter ativas as informações que lê, enquanto busca e ativa informações correlatas em sua base de conhecimento e estabelece nexos entre elas.

Para além da fácil previsibilidade lógica da importância da memória de trabalho para a compreensão leitora, as evidências empíricas se acumulam. Diferenças individuais na MT implicam diferenças na compreensão em adultos e crianças (Linderholm \& van den Broek, 2002) e são preditoras do desenvolvimento da compreensão em crianças (Sesma, Mahone, Levine, Eason, \& Cutting, 2009).

Há ainda evidências mais diretas de que pelo menos parte do efeito da MT sobre a compreensão incide, exatamente, sobre a implementação da capacidade de fazer inferências, assim como sobre a capacidade de auto-monitorização (Engle \& Conway, 1988).

\section{Planejamento}

Ao elencar as características que diferenciam bons de maus compreendedores, Kaufman (2010) destacou que os primeiros (mas não os outros) abordam o texto e dele se apropriam, de modo estratégico: (1) desenvolvem objetivos e planos mesmo antes de começar a leitura; (2) empregram uma série de estratégias de manutenção das informações enquanto leem, tais como tomar notas e marcar o texto; (3) se fazem perguntas sobre o que lêem, examinando a coerência do texto com as informações disponíveis em sua base de conhecimentos; (4) lêem nas entrelinhas, fazendo inferências e, ao final da leitura (5) refletem sobre os elementos factuais e conceituais do texto e os integram, modificando seus esquemas. Em suma, assim como já evidenciado por outros (RAND Reading Study Group, 2002), bons leitores abordam o texto de forma estratégica, planejada e nada incidental.

A importância crucial do planejamento para a compreensão na leitura é endossada pela observação de comprometimento desta função executiva, inclusive em tarefas não verbais, em crianças com dificuldade específica de compreensão leitora (Cutting, Materek, Cole, Levine, \& Mahone, 2009; Locascio, Mahone, Eason, \& Cutting, 2010; Sesma et al., 2009). Indicação mais direta da relação causal entre planejamento e compreensão é trazida por estudos como o de Eason, Goldberg, Young, Geist e Cutting (2012). Estes autores mostraram que ao se deparar com textos mais complexos e longos, indivíduos com déficits na capacidade de planejamento e organização, não desenvolvem estratégias eficientes de abordagem da leitura. Samuelstuen e Braten (2005) também identificaram o uso de estratégias de organização, elaboração e monitorização como fortes preditores da compreensão leitora.

Evidências mais indiretas da participação dessa função executiva na compreensão advém da eficácia de intervenções que capitalizam no aumento do controle executivo do processo de compreensão, seja ensinando a usar a estrutura de um texto narrativo como estruturante para a compreensão dos seus elementos e das relações entre eles (p. ex. Beck \& McKeown, 1981; Idol \& Croll, 1987; Pearson, 1982), seja ensinando a buscar pistas no texto que auxiliem na compreensão de uma palavra (McGee \& Johnson, 
2006, por exemplo). Nesses estudos, pode-se dizer que um método, uma estratégia, um plano de ação de como abordar o texto, é ensinado aos sujeitos.

\section{Controle inibitório}

O controle inibitório se refere a capacidade de controlar a própria atenção, comportamentos, pensamentos e emoções, em detrimento de fortes predisposições internas ou da força dos estímulos externos (Diamond, 2013). Para alguns autores, constitui uma das funções executivas mais primárias e que, inclusive, antecede as outras em termos de trajetória de desenvolvimento e as influencia e modula diretamente (Diamond, 2013).

O papel da capacidade de inibir respostas a estímulos não relevantes a atividade em curso, na compreensão leitora também é logicamente compreensível. Selecionar as informações mais importantes do texto, para que seja possível estabelecer nexos entre elas, requer a capacidade de inibir o efeito de informações não cruciais. Um leitor cuja atenção é capturada por qualquer estimulo forte, poderia ter dificuldades nesse processo. Além disso, este leitor poderia ter dificuldade até na seleção de informações na sua própria base de conhecimentos. A exposição a um dado tema sempre ativa toda uma rede de informações a ele relacionadas, na base de conhecimentos do sujeito, o que pode atrapalhar aquele que tem dificuldade de selecionar e inibir o que é menos importante em dado momento (Rapp et al., 2007).

De fato, as evidências empíricas conspiram nesta direção. Diferenças individuais em termos de inibição estão correlacionadas com diferenças na compreensão. Crianças com problemas de compreensão apresentaram dificuldade de eliminar informações não importantes da memória de trabalho e da memória de longo prazo, durante a compreensão na leitura. E, mesmo quando instadas a ignorar certas palavras enquanto liam, crianças com pobre controle inibitório não conseguiam fazê--lo (Cain, 2006). Gernsbacher e Faust (1991) verificaram que entre leitores adultos, os piores compreendedores tem mais dificuldade de inibir significados inapropriados de palavras ambíguas e de homófonos, do que melhores compreendores.

\section{Alocação de atenção}

O comprometimento da habilidade de adaptar os recursos atencionais e de processamento às demandas da tarefa em curso tem sido apontado como fonte de problemas na compreensão leitora (Liu, Reichle \& Gao, 2013). Van den Broek, Helder e Van Leijenhorst (2013) observaram dificuldade de compreensão quando o desenvolvimento da sensitividade a centralidade estrutural do texto se encontra comprometido. Esta sensitividade se refere a capacidade de focalizar nos aspectos estruturalmente centrais na representação mental do texto. Indivíduos com dificuldade na alocação de atenção são facilmente distraídos por detalhes enquanto lêem, especialmente se os textos são mais longos (Long, Seely, \& Oppy, 1997)

\section{O processo de compreensão}

O produto da compreensão leitora é a construção de uma representação da informação textual na mente do leitor, ao final do processo. Quando há compreensão, esta representação é coerente, isto é, foram estabelecidas, em grau suficiente, conexões apropriadas e significativas entre os elementos do texto e entre estes e a base de conhecimentos do leitor, de forma a produzir um todo integrado (Kintch, 1988). Esta representação constitui a base para ações futuras relacionadas ao texto, como recontar, responder perguntas ou empregar as informações do texto para algum fim (van den Broek \& Espin, 2012).

Para chegar a este produto um processamento complexo é necessário. Os processos de construção de coerência durante a leitura refletem um equilíbrio entre a tentativa do leitor de criar coerência (atendendo a um standard de coerência) e a qualidade das funções cognitivas necessárias ao longo da leitura. $\mathrm{O}$ standard de coerência do leitor se refere ao grau de coerência semântica que ele visa manter durante a leitura. É afetado pelo tipo de texto e pelas características da pessoa como, por exemplo seu nível de funcionamento cognitivo global, seu interesse pelo texto, ou a finalidade da leitura (Van den Broek, BohnGettler, Kendeou, Carlson, \& White, 2011).

Para a ACCL, a construção de coerência constitui um processo dinâmico, no qual são recrutados, em vários momentos e ciclicamente, os processos inferenciais automáticos e estratégicos, sendo balanceado em 
todo o seu percurso pelo standard de coerência do leitor (Rapp et al., 2007).

O leitor se engajará em um processamento inferencial apropriado para atender a um standard de coerência, dentro, é claro, dos limites impostos por seu funcionamento cognitivo, tanto em termos de processos de baixo quanto de alto nível (van den Broek, Rapp, \& Kendeou, 2005). Assim, em alguns momentos o recrutamento de processos inferenciais automáticos poderá ser suficiente para atender ao standard de coerência do leitor, enquanto que em outros, esta ativação apenas trará maior desequilíbrio em termos de coerência, de forma a tornar necessários processsos inferenciais estratégicos. Por exemplo, diante de uma aparente contradição entre o que foi lido antes no texto e o que está sendo lido agora, o leitor precisará rever partes anteriores do texto ou recorrer a sua base de conhecimento na busca de coerência. Nesse sentido o standard de coerência afeta inclusive o recrutamento de diferentes processos inferenciais ao longo da leitura, podendo afetar a qualidade do produto final, a despeito da integridade funcional dos referidos processos.

Assim, para a ACCL, o alcance da compreensão leitora depende das capacidades do leitor, em termos da integridade e potencialidade das funções cognitivas recrutadas ao longo do processo (desde a decodificação até a construção da representação do texto), mas também depende em alto grau do que o leitor espera compreender do texto (seu standard de coerência). A intenção de compreensão do leitor é trazida para dentro do modelo teórico, como variável determinante do processo de compreensão leitora e de seu produto.

\section{IMPLICAÇÕES DA ACCL PARA A EDUCAÇÃO E PARA A PRÁTICA CLÍNICA}

\section{1) Estimulação de funções cognitivas cruciais para a compreensão leitora na pré-escola}

O modelo de compreensão leitora proposto na ACCL aponta para a continuidade entre o desenvolvimento da compreensão na oralidade e na leitura e identifica as funções executivas e a capacidade de fazer inferências como fundamentais para a compreensão. Assim, aponta para a importância da estimulação dessas funções antes mesmo da criança ser alfabetizada.

Não é novidade a importância do desenvolvimento da linguagem oral, em termos de vocabulário, gramá- tica, sintaxe, expressão e compreensão, para o desenvolvimento das habilidades de decodificação. Embora a pré-escola não seja um preparatório para o ensino fundamental, as escolas incluem em suas práticas atividades para estimular o desevolvimento dessas funções e muitas incluem até atividades que estimulam o desenvolvimento do controle motor fino e das habilidades de consciência fonológica.

O modelo de compreensão leitora proposto na ACCL sugere a participação de vários processos cognitivos, linguísticos e não lingüísticos, nessa habilidade, e em particular das funções executivas e da capacidade de fazer inferências. Tendo em vista que estas funções não são exclusivas da escrita e seu desenvolvimento começa cedo na infância, seria interessante poder incluir a sua estimulação na pré-escola utilizando outras mídias além do texto escrito.

Kendeou et al. (2005), discorrendo sobre esta possibilidade, aponta para o uso de televisão e apresentação oral de histórias para crianças mais novsa. Além disso, discute os tipos de intervenção que poderiam ser interessantes para desenvolvimento da compreensão. Os autores indicam metodologias que capitalizam no desenvolvimento das funções executivas no contexto da compreensão oral, como o uso de questionamento com perguntas específicas que ajudem a compreensão da cadeia causal da história e que levem em conta os níveis de inferência possíveis para as crianças em diferentes idades.

Outros autores já criaram métodos para estimulação do desenvolvimento das funções executivas baseados na psicologia cognitiva que poderiam também ser aplicados no contexto da pré-escola (Diamond, 2012; Dias \& Seabra 2013) e assim contribuir para o desenvolvimento da compreensão da leitura, mais tarde.

\section{2) Implicações para a prática clínica: avaliação e remediação}

A concepção de que a compreensão leitora depende da participação de processos de baixo nível, como a decodificação, e de processos de mais alto nível como as diferentes funções executivas e a capacidade de fazer inferências, implica na idéia de que dificuldades de compreensão podem ter diferentes causas. 
Por exemplo, uma criança pode ter dificuldade de compreensão porque a decodificação não se desenvolveu a contento e, portanto, "rouba" recursos atencionais fundamentais para o funcionamento executivo. Mesmo que a criança tenha um bom desenvolvimento destas últimas funções, pode não conseguir atualizar esta capacidade em desempenho, exatamente porque os recursos atencionais estão sendo drenados pelos processos de baixo nível (Rapp et al., 2007).

Outra criança pode ter dificuldade de compreender o que lê, estando a decodificação perfeitamente desenvolvida. Isto pode decorrer de comprometimento no controle inibitório (Gernsbacher \& Faust, 1991; Kieffer, Vukovic, \& Berry, 2013), uma função de alto nível. Assim, ao ler, a criança o faz com fluência, mas sua atenção é capturada por determinadas palavras ou frases do texto que não pertencem a cadeia causal principal e levam, por exemplo, à geração de hipóteses equivocadas quanto ao que está sendo dito no texto.

Em outro caso, a dificuldade de compreensão pode ocorrer, estando a decodificação preservada, porque a criança apresenta uma demora no desenvolvimento da função executiva de planejamento. E, neste caso, embora as outras funções executivas estejam preservadas, a criança não tem a iniciativa de construir hipóteses a respeito de relações entre partes do texto e entre estas partes e sua base de conhecimentos, apresentando dificuldade em recrutar processos inferenciais estratégicos. Não se faz perguntas durante a leitura, ou o faz de forma muito restrita, e esta embora fluente, leva quando muito, a compreensão de informações literalmente declaradas no texto (Eason et al., 2012; Gersten, Fuchs, Williams, \& Baker, 2001).

Ainda, uma outra criança pode ter uma limitação na capacidade da memória de trabalho e, apesar de todas as outras funções estarem preservadas, ter dificuldade em fazer inferências porque não consegue manter e operar mentalmente com quantidades de informação necessárias para a compreensão (Just \& Carpenter, 1992; Cain, Oakhill, \& Bryant, 2004).

Outra possibilidade é que uma criança que dispõe de processos cognitivos de baixo e alto nível íntegros e bem desenvolvidos, pode ter dificuldade para compreender um texto, em função de fatores motivacionais que afetem o seu standard de compreensão e, em decorrência, leve a falhas no recrutamento dos processos inferenciais necessários à compreensão.
São vários os perfis possíveis, mas não se pode deixar de considerar ainda um outro, em que a criança tenha dificuldades de compreensão (estando os processos de decodificação preservados) em decorrência de restrições do funcionamento executivo e da base de conhecimentos (Rapp et al., 2007).

A ideia de que vários processos cognitivos estão envolvidos na compreensão leitora, incluindo processos lingüísticos e não linguísticos, implica na existência de múltiplos perfis cognitivos possíveis em casos de dificuldade de compreensão, mesmo quando a decodificação está preservada. Esta observação tem implicações para a prática clínica em termos de avaliação e remediação. A localização precisa da fonte (ou das fontes) de dificuldade possibilita intervenções mais precisas e baseadas em evidências (Rapp et al., 2007; Kendeou et al., 2014; McMaster, Espin, \& van den Broek, 2014).

\section{3) Prática clínica: vias alternativas de estimulação}

A indicação de continuidade entre oralidade e leitura no desenvolvimento da capacidade de compreensão (Gernsbacher, 1991; Kendeou et al., 2005/2008; Kendeou, van den Broek, White, \& Lynch, 2007; Kendeou et al., 2014), sugere a possibilidade do uso de protocolos de estimulação no campo da oralidade buscando efeitos na compreensão leitora.

Esta via alternativa pode ser muito interessante na prática clínica, tendo em vista que possibilita evitar alguns obstáculos freqüentes. Crianças com dificuldade de compreensão muitas vezes apresentam comportamento de esquiva diante da leitura, o que compromete a estimulação. Além disso, muitas vezes a dificuldade de compreensão decorre de dificuldades de decodificação combinadas com dificuldades de mais alto nível. Nesses casos a leitura pode se tornar penosa, comprometendo a estimulação das habilidades de alto nível.

\section{OBSERVAÇÕES FINAIS}

A importância da compreensão leitora é indiscutível, visto sua função instrumental na vida acadêmica e na inserção no mundo. As publicações nacionais sobre o tema ainda ocorrem em número bastante modesto, em vista da restrição apresentada pelos brasileiros em termos desta habilidade. Este artigo apresentou a abordagem cognitiva da compreensão leitora. Tal 
teoria considera a participação de funções cognitivas de baixo e alto nível na compreensão na leitura, de forma semelhante a outros modelos téoricos. No entanto, se diferencia pela ênfase nas funções executivas e pelo detalhamento do processo de compreensão.

Estas características do modelo resultam em implicações importantes para a educação e para a prática clínica com crianças com dificuldade de compreensão leitora. Por exemplo, na medida em que as funções executivas e os processos inferenciais não são específicos à compreensão leitora, sua estimulação pode anteceder a alfabetização e, talvez, contribuir para que esta se desenvolva de forma apropriada. No que tange a prática clínica, conhecer o papel das diferentes funções executivas no complexo processo de compreensão possibilita precisar que elementos do perfil cognitivo podem estar causando a dificuldade de compreensão. Isto incrementaria a precisão no delineamento de estratégias de remediação. A abordagem cognitiva da compreensão leitora pode trazer constribuições e novas perguntas não apenas para a prática clínica e a educação, mas também para a pesquisa.

\section{REFERÊNCIAS}

Ackerman, B. P. (1988). Reason inferences in the story comprehension of children and adults. Child Development, 59(5) 1426-1442.

Ackerman, P. L. (1988). Determinants of individual differrences during skill acquisition: Cognitive abilities and information processing. Journal of Experimental Psychology: General, 117(3), 288-318.

Baddeley, A. (2003). Working memory and language: An overview. Journal of Communication Disorders, 36(3), 189-208.

Baddeley, A. D., \& Hitch, G. J. (1994). Developments in the concept of working memory. Neuropsychology, 8(4), 485-493.

Beck, I. L., \& McKeown, M. G. (1981). Developing questions that pro-mote comprehension: The story map. Language Arts, 58, 913-918.

Bishop, D. V., \& Snowling, M. J. (2004). Developmental dyslexia and specific language impairment: same or different? Psychological Bulletim, 130, 858-886.

Brasil (2009). PISA 2009 - Program for international Student Assessment: resultados preliminares. Brasília: Instituto Nacional de Estudos e Pesquisas Educacionais Anísio Teixeira (INEP), Ministério da Educação (MEC). Retrieved from: http://www.inep.gov.br/internacional/pisa

Brasil (2012). PISA 2012 - Program for international Student Assessment: resultados preliminares. Brasília: Insti- tuto Nacional de Estudos e Pesquisas Educacionais Anísio Teixeira (INEP), Ministério da Educação (MEC). Retrieved from: http://www.inep.gov.br/internacional/pisa

Brown, A. L., Palincsar, A. S., \& Armbruster, B. B. (1984). Instructing comprehension-fostering activities in interactive learning situations. Learning and comprehension of text, 255-286.

Cain, K., \& Oakhill, J. (2006). Profiles of children with specific reading comprehension difficulties. British Journal of Educational Psychology, 76, 683-696.

Cain, K., \& Oakhill, J. V. (1999). Inference making ability and its relation to comprehension failure in young children. Reading and Writing, 11(5-6), 489-503.

Cain, K., Oakhill, J., \& Bryant, P. (2000). Phonological skills and comprehension failure: A test of the phonological processing deficit hypothesis. Reading and Writing, 13(1-2), 31-56.

Cain, K., Oakhill, J., \& Bryant, P. (2004). Children's reading comprehension ability: Concurrent prediction by working memory, verbal ability, and component skills. Journal of Educational Psychology, 96(1), 31-42.

Capellini, S. A., Pinto, C. D. A. R., \& Cunha, V. L. O. (2015). Reading Comprehension Intervention Program for Teachers from 3rd Grade'Students.Procedia-Social and Behavioral Sciences, 174, 1339-1345.

Capellini, S. A., Santos, B. dos, \& Uvo, M. F. C. (2015). Metalinguistic Skills, Reading and Reading Comprehension Performance of Students of the 5th Grade. Procedia - Social and Behavioral Sciences, 174, 1346-1350.

Clarke, P., Snowling, M., Truelove, E., \& Hulme, C. (2010). Ameliorating children's reading comprehension difficulties: A randomized controlled trial. Psychological Science, 21, 1106-1116.

Corso, H. V., Sperb, T. M., \& Salles, J. F. de. (2013). Leitura de palavras e de texto em crianças: efeitos de série e tipo de escola, e dissociações de desempenhos. Letras de Hoje, 48(1), 81-90.

Cutting, L.E. Materek, A., Cole, C.A.S., Levine, T., \& Mahone, E. M. (2009). Effects of language, fluency, and executive function on reading comprehension performance. Annals of Dyslexia. 59, 34-54.

Diamond, A. (2012). Activities and programs that improve children's executive functions. Current Directions in Psychological Science, 21(5), 335-341.

Diamond, A. (2013). Executive functions. Annual review of psychology, 64, 135-168.

Dias, N. M., \& Seabra, A. G. (2013). Programa de intervenção sobre a autorregulação e funções executivasPIAFEx. São Paulo: Memnon.

Eason, S. H., Goldberg, L. F., Young, K. M., Geist, M. C., \& Cutting, L. E. (2012). Reader-text interactions: How differential text and question types influence cognitive skills needed for reading comprehension. Journal of Educational Psychology, 104(3), 515-528. 
Engle, R. W., \& Conway, A. R. A. (1998). Working memory and comprehension. In R. H. Logie \& K. J. Gilhooly (Eds.), Working memory and thinking (pp. 67-92). East Sussex, UK: Psychology Press.

Ferreira, S. P. A., \& Dias, M. da G. B. B. (2002). Compreensão de leitura: estratégias de tomar notas e da imagem mental. Psicologia: Teoria E Pesquisa, 18(1), 51-62.

Gernsbacher, M. A., \& Faust, M. E. (1991). The mechanism of suppression: a component of general comprehension skill. Journal of Experimental Psychology. Learning, Memory, and Cognition, 17(2), 245-262.

Gersten, R., Fuchs, L. S., Williams, J. P., \& Baker, S. (2001). Teaching Reading Comprehension Strategies to Students With Learning Disabilities: A Review of Research. Review of Educational Research, 71(2), 279320.

Gomes, M. A. M., \& Boruchovitch, E. (2011). Aprendizagem autorregulada da leitura: resultados positivos de uma intervenção psicopedagógica. Psicologia: teoria pesquisa, 27(3), 291-299.

Gough, P. B., \& Tunmer, W. E. (1986). Decoding, Reading, and Reading Disability. Remedial and Special Education, 7(1), 6-10.

Guimarães, P. M. C. S., de Abreu Emmerick, T., Vicente, A. L., \& Soares, A. B. (2012). Diagnóstico da compreensão textual de alunos de $4^{\circ}$ e $5^{\circ}$ anos do Ensino Fundamental. Psicologia Escolar e Educacional, 16(1), 95103.

Hansen, J., \& Pearson, P. D. (1983). An instructional study: Improving the inferential comprehension of good and poor fourth-grade readers. Journal of Educational Psychology, 75(6), 821.

Idol, L., \& Croll, V. J. (1987). Story-mapping training as a means of improving reading comprehension. Learning Disability Quarterly, 10, 214-229.

Joly, M. C. R. A., \& Piovezan, N. M. (2012). Avaliação do Programa Informatizado de Leitura Estratégica para estudantes do ensino fundamental. Paidéia (Ribeirão Preto), 22(51), 83-90.

Just, M. A., \& Carpenter, P. A. (1992). A capacity theory of comprehension: individual differences in working memory. Psychological Review, 99(1), 122-149.

Kaufman, C. (2010). Executive function in the classroom. Baltimore: Brookes Publishing.

Kendeou, P., Bohn-Gettler, C., White, M. J., \& van den Broek, P. (2008). Children's inference generation across different media. Journal of Research in Reading, 31(3), 259-272.

Kendeou, P., Lynch, J., Broek, P., Espin, C., White, M., \& Kremer, K. (2005). Developing successful readers: Building early comprehension skills through television viewing and listening. Early Childhood Education Journal, 33(2), 91-98.
Kendeou, P., van den Broek, P., Helder, A., \& Karlsson, J. (2014). A Cognitive View of Reading Comprehension: Implications for Reading Difficulties. Learning Disabilities Research \& Practice, 29(1), 10-16.

Kendeou, P., van den Broek, P., White, M., \& Lynch, J. (2007). Preschool and early elementary comprehension: Skill development and strategy interventions. In D. S. McNamara (Ed.) Reading comprehension strategies: Theories, interventions, and technologies (pp. 27-45). Mahwah, NJ: Erlbaum.

Kieffer, M. J., Vukovic, R. K., \& Berry, D. (2013). Roles of Attention Shifting and Inhibitory Control in FourthGrade Reading Comprehension. Reading Research Quarterly, 48(4), 333-348.

Kintsch, W. (1998). Comprehension: A Paradigm for Cognition. New York: Cambridge University Press.

Kintsch, W., \& Van Dijk, T. A. (1978). Toward a model of text comprehension and production. Psychological Review, 85(5), 363-394.

Linderholm, T., \& van den Broek, P. (2002). The effects of reading purpose and working memory capacity on the processing of expository text. Journal of Educational Psychology, 94(4), 778.

Linderholm, T., Virtue, S., Tzeng, Y., \& van den Broek, P. (2004). Fluctuations in the availability of information during reading: Capturing cognitive processes using the landscape model. Discourse Processes, 37(2), 165-186.

Liu, Y., Reichle, E. D., \& Gao, D.-G. (2013). Using Reinforcement Learning to Examine Dynamic Attention Allocation During Reading. Cognitive Science, 37(8), 1507-1540.

Locascio, G., Mahone, E. M., Eason, S., \& Cutting, L. (2010). Executive dysfunction among children with reading comprehension deficits. Journal of learning disabilities.10 (5), 1-14.

Long, D. L., Seely, M. R., \& Oppy, B. J. (1997). Individual differences in readers' sentence- and text-level representations. Journal of Memory and Language, 36, 129-145.

Lorch Jr, R. F., \& van den Brock, P. (2000). Understanding Reading Comprehension. Psychology of Education: The school curriculum, 3, 186.

Machado, M. A. R. (2010). Compreensão de leitura: o papel do processo inferencial. Anápolis, GO: Editora da Universidade Estadual de Goiás.

Magliano, J. P., Wiemer-Hastings, K., Millis, K. K., MuÑoz, B. D., \& Mcnamara, D. (2002). Using latent semantic analysis to assess reader strategies. Behavior Research Methods, Instruments, \& Computers, 34(2), 181188.

McGee, A., \& Johnson, H. (2006). The effect of inference training on skilled and less skilled comprehenders. Educational Psychology, 23, 49-59. 
McMaster, K. L., Espin, C. A., \& Broek, P. (2014). Making connections: Linking cognitive psychology and intervention research to improve comprehension of struggling readers. Learning Disabilities Research \& Practice, 29(1), 17-24.

Miyake, A., Friedman, N. P., Emerson, M. J., Witzki, A. H., Howerter, A., \& Wager, T. D. (2000). The unity and diversity of executive functions and their contributions to complex "Frontal Lobe" tasks: a latent variable analysis. Cognitive Psychology, 4l(1), 49-100.

O’Brien, E. J., \& Albrecht, J. E. (1992). Comprehension strategies in the development of a mental model. Journal of Experimental Psychology. Learning, Memory, and Cognition, 18(4), 777-784.

Oakhill, J. \& Yuill, N. (1996). Higher order factors in comprehension disability: Processes and remediation. In C. Cornoldi \& J. Oakhill (Eds.), Reading comprehension difficulties. Mahwah: Erlbaum.

Oakhill, J. (1994). Individual differences in children's text comprehension. In Gernsbacher, Morton Ann (Ed). Handbook of psycholinguistics (pp. 821-848). San Diego: Academic Press.

Oakhill, J., Cain, K., \& Bryant, P. E. (2003). The dissociation of word reading and text comprehension: Evidence from component skills. Language and Cognitive Processes, 18(4), 443-468.

Oliveira, R. M. (2015). Intervenções para melhorar dificuldade específica de compreensão leitora. In Alves, L. M.; Mousinho, R. \& Capellini, S. A. (Org.), Dislexia: Novos temas, novas perspectivas (pp. 219-232). Rio de Janeiro: Wak.

Parente, M. A. de M. P., Holderbaum, C. S., Virbel, J., \& Nespoulous, J.-L. (2005). Question-asking organization. Psicologia: Reflexão E Crítica, 18(2), 267-276.

Paris, S. G., Cross, D. R., \& Lipson, M. Y. (1984). Informed Strategies for Learning: A program to improve children's reading awareness and comprehension. Journal of Educational psychology, 76(6), 1239.

Pearson, P. D. (1982). Asking questions about stories. Ginn occasional papers, 16, 1-11.

Perfetti, C. (2007). Reading ability: Lexical quality to comprehension. Scientific studies of reading, 11(4), 357-383.

RAND Reading Study Group. (2002). Reading for understanding; Toward an R\&D program in reading comprehension. Santa Monica: RAND.

Rapp, D. N., van den Broek, P., McMaster, K. L., Kendeou, P., \& Espin, C. A. (2007). Higher-order comprehension processes in struggling readers: A perspective for research and intervention. Scientific Studies of Reading, 11, 289-312.

Salles, J.F., \& Parente, M.A.M.P. (2004). Compreensão textual em alunos de segunda e terceira séries: uma abordagem cognitiva. Estudos e Pesquisas em Psicologia, 9(1), 71-80.
Samuelstuen, M. S., \& Bråten, I. (2005). Decoding, knowledge, and strategies in comprehension of expository text. Scandinavian Journal of Psychology, 46(2), 107117.

Santos, A. A. A. dos, \& Oliveira, E. Z. de. (2010). Assessment and development of reading comprehension with elementary school students. Psico-USF, 15(1), 81-91.

Sesma, H. W., Mahone, E. M., Levine, T., Eason, S. H., \& Cutting, L. E. (2009). The contribution of executive skills to reading comprehension. Child Neuropsychology, 15(3), 232-246.

Spinillo, A., \& Hodges, L. (2012) Análise de Erros e Compreensão de Textos: comparações entre diferentes situações de leitura. Psicologia: Teoria e Pesquisa, 28(4), 381-388.

Van den Broek, P. (1997). Discovering the cement of the universe: The development of event comprehension from childhood to adulthood. In P. van den Broek, P. W. Bauer \& T. Bourg (Eds.), Developmental spans in event comprehension and representation (pp. 321-342). Mahwah, NJ: Lawrence Erlbaum Associates.

Van den Broek, P. (2010). Using texts in science education: Cognitive processes and knowledge representation. Science, 328(5977), 453-456.

Van den Broek, P., \& Espin, C. A. (2012). Connecting cognitive theory and assessment: Measuring individual differences in reading comprehension. School Psychology Review, 41, 315-325.

Van den Broek, P., Bohn-Gettler, C., Kendeou, P., Carlson, S., \& White, M. J. (2011). When a reader meets a text: The role of standards of coherence in reading comprehension. Text relevance and learning from text, 123-140.

Van den Broek, P., Helder, A., \& Van Leijenhorst, L. (2013). Sensitivity to structural centrality: Developmental and individual differences in reading comprehension skills. In M. A. Britt, S. R. Goldman, \& J.-F. Rouet (Eds.), Reading: From words to multiple texts (pp. 132146). New York: Routledge.

Van den Broek, P., Rapp, D., \& Kendeou, P. (2005). Integrating memorybased and constructionist processes in accounts of reading comprehension. Discourse Processes, 39(2), 299-316.

Van den Broek, P.W., (1994) Comprehension and memory of narrative texts: Inferences and coherence. In Gernsbacher, M.A. (Ed.), Handbook of psycholinguistics, 539588. New York: Academic Press.

Yuill, N., \& Joscelyne, T. (1988). Effect of organizational cues and strategies on good and poor comprehenders' story understanding. Journal of educational Psychology, 80(2), 152. 